\title{
A Cross-Layer Location-Based Approach for Mobile-Controlled Connectivity
}

\author{
T. Inzerilli, ${ }^{1}$ A. M. Vegni, ${ }^{2}$ A. Neri, ${ }^{2}$ and R. Cusani ${ }^{3}$ \\ ${ }^{1}$ Dipartimento per le Comunicazioni, I.S.C.T.I., Ministero dello Sviluppo Economico, Viale America 201, 00144 Rome, Italy \\ ${ }^{2}$ Department of Applied Electronics, University of Roma Tre, Via della Vasca Navale 84, 00146 Rome, Italy \\ ${ }^{3}$ Department of Information Engineering, Electronics and Telecommunications (DIET), University of Rome "Sapienza", \\ Via Eudossiana 18, 00184 Rome, Italy \\ Correspondence should be addressed to A. M. Vegni, amvegni@uniroma3.it
}

Received 2 July 2010; Revised 5 November 2010; Accepted 3 December 2010

Academic Editor: Stefania Colonnese

Copyright ( $) 2010$ T. Inzerilli et al. This is an open access article distributed under the Creative Commons Attribution License, which permits unrestricted use, distribution, and reproduction in any medium, provided the original work is properly cited.

We investigate into the potentiality of an enhanced Power and Location-based Vertical Handover (PLB-VHO) approach, based on a combination of physical parameters (i.e., location and power attenuation information), for mobile-controlled connectivity across UMTS and WLAN networks. We show that the location information in a multiparameter vertical handover can significantly enhance communication performance. In the presented approach a power attenuation map for the visited area is built and kept updated by exploiting the information sharing of power measurements with other cooperating mobile devices inside the visited networks. Such information is then used for connectivity switching in handover decisions. The analytical model for the proposed technique is first presented and then compared with a traditional Power-Based approach and a simplified Location-Based technique. Simulation results show the effectiveness of PLB-VHO approach, in terms of (i) network performance optimization and (ii) limitation of unnecessary handovers (i.e., mitigation of ping-pong effect).

\section{Introduction}

Current heterogeneous wireless networking scenarios include multimode Mobile Terminals (MTs) equipped with multiple wireless Network Interface Cards (NICs) and providing Vertical Handover (VHO) capability to autonomously select the best access. VHO allows switching from one access technology to another thus offering additional functionalities with respect to horizontal handover, where MTs move from an Access Point (AP) to another without changing the serving access network $[1,2]$.

A VHO process aims to guarantee seamless connectivity between heterogeneous wireless networks inside areas where simultaneous coverage from multiple networks is provided $[3,4]$. Selection of the serving network can be based on optimality criteria which balance different factors including, for instance, monetary cost, energy consumption and enduser Quality-of-Service (QoS) [5]. In order to obtain an optimal tradeoff among these factors, while assuring high service continuity, fast and reliable procedures for the selection of the serving network have to be designed in the case of link degradation or loss of connectivity.

Various wireless networks exhibit quite different data rates, link errors, transmission range and transport delay. As a consequence, a direct comparison between heterogeneous wireless links in order to select the best network to attach to is not always straightforward. In general, a VHO strategy requires a preliminary definition of performance metrics for all the networks providing access in a visited environment, in order to compare the QoS offered by each of them and to decide for the best one $[2,5]$.

VHO decisions can be based on wireless channel state, network layer characteristics, as well as on application requirements when required. In this respect, various parameters can be taken into account, such as the type of the application (e.g., conversational, streaming, interactive, background), minimum bandwidth, tolerable maximum delay, bit error rate, transmitting power, current MT battery status, as well as user's preferences $[1,5]$. 
In this paper is presented a novel mobile-controlled $\mathrm{VHO}$ scheme, in the following denoted as PLB-VHO. PLB-VHO is based on an integrated approach using two main procedures, that is, (i) a Distributed Attenuation Map Building (DAMB) and (ii) an Enhanced Location-based (ELB) VHO based on the calculated attenuation maps. PLB-VHO aims to select the best wireless access network in real-time and improve the overall network performance.

The paper is structured as follows. In Section 2 basic concepts on vertical handover mechanism are discussed. Section 3 describes the main procedures of the proposed PLB-VHO, that is, Distributed Attenuation Map Building and Enhanced Location-based VHO. Section 4 is dedicated to a performance comparison between the proposed PLBVHO approach with a Power-Based VHO technique (i.e., $\mathrm{PB}-\mathrm{VHO}$ ) and a simplified Location-Based VHO solution (i.e., LB-VHO, where no attenuation map is considered for vertical handover decisions). Finally, conclusions are drawn in Section 5.

\section{Related Work}

In this section we propose an overview of the main criteria behind a vertical handover mechanism and describe how our technique results are innovative with respect to the related works.

Handover procedures typically fall into two main classes Mobile-Controlled Handover (MCHO) and NetworkControlled Handover ( $\mathrm{NCHO}$ ), depending on whether a handover is both initiated and controlled either by the MT or by the network, respectively [6]. In horizontal handover management, $\mathrm{MCHO}$ is the most common case, especially for WLAN environments, while NCHO is generally the preferred choice for cellular networks where resource optimization and load management are centralized. When a handover across different networks is considered-Vertical Handover-the MCHO appears a more practical approach, as handover decisions do not require interaction between heterogeneous networks and distinct network providers, which should interact to perform a handover across the two networks. In this work we are then focusing on an $\mathrm{MCHO}$ approach.

Furthermore, VHO schemes can be classified on the basis of the criteria and parameters adopted for initiating a handover from a Serving Network (SN) to a new Candidate Network (CN). Namely, we can enlist the following main schemes differing on the metrics driving handover decisions:

(1) Received Signal Strength- (RSS-) based VHO algorithms: this technique is native for horizontal handover mechanisms, specially for GSM system, which mainly considers RSS as the only decision metric. A drop of the RSS below the receiver sensitivity denotes lack of connectivity, which necessarily requires the execution of a handover [7-9]. The evolution of criteria for handover decisions provides for cost of services, power consumption, Quality-of-Service, positioning, and velocity of the mobile terminal, which are mainly oriented to maximize user requirements;

(2) Signal-to-Noise and Interference ratio- (SINR-) based VHO algorithms: SINR directly impacts achievable goodput in a wireless access network [10,11]. (The goodput [Bps] defines the amount of useful information per second. Such definition does not consider dropped packets or packet retransmissions, as well as protocol overhead.) In some wireless access technologies the transmission rate is dynamically adapted to the channel state condition. For instance, in the HSDPA network the modulation and the channel coding schemes vary versus the measured SINR, and both schemes influence the actual communication goodput;

(3) multiparameter QoS-based VHO algorithms: VHO algorithms can be based on the overall quality assessment for the available networks obtained by balancing various parameters $[12,13]$. In order to improve the perceived communication quality, these approaches mostly consider the user connectivity to be switched to a candidate network, whenever the bandwidth is higher than the currently experienced in the actual serving network;

(4) speed-based VHO algorithms: in vehicular environments where users move fast at different speeds, seamless connectivity results in a challenging issue. Handovers should be performed on the basis of specific factors as vehicle mobility pattern and locality information, rather than currently perceived QoS, which might change rapidly over time. Speed-based $\mathrm{VHO}$ approaches combine the user location information, the measured vehicle speed, and the currently perceived QoS to make predictions and perform handovers [14]. In [15] whenever the vehicle speed falls below a given threshold, a vertical handover is executed in order to maintain a required QoS level and a seamless connectivity;

(5) location-based VHO algorithms: the location information can drive handover initiation allowing an MT to select that network which currently assures the best performance-network QoS parameterson the basis of MT proximity to the nearest AP $[16,17]$. In general, a location-based VHO technique consists in a preliminary handover initiation phase triggered on the basis of MT's location; handover is then carried out by following a QoS estimation phase. For instance, in [18] the MT's location information is exploited to initiate a goodput assessment, followed, if necessary, by a vertical handover execution. In [19] the prototype of a dual mode UMTS-WLAN mobile terminal for seamless connectivity and locationaware service is presented.

Notice that in general a vertical handover mechanism is oriented to maintain a seamless connectivity and limit the well-known ping-pong effect [20]. Unwanted and unnecessary vertical handovers often occur, especially when a 
mobile terminal moving back and forth between the two neighboring wireless networks - or in general around a corner that involves three or more wireless networkstriggers handover attempts repeatedly. This leads to excessive location and registration updates (i.e., network resource consumption), frequent connectivity interruptions, as well as seriously affection to mobile terminal's QoS (i.e., decreasing battery life). Typically, the ping-pong effect is a consequence of fluctuations of signal strength received by the mobile terminal from adjacent cells, causing unwanted handovers between neighboring wireless networks.

In addition, from the user perspective, frequent handovers can be experienced as numerous unpleasant transients of service interruption, while they might also produce excessive battery consumption. It follows that the minimization of the number of VHOs is a key issue in handover management $[8,9,11,14,15,18,20,21]$. For this purpose, many handover algorithms incorporate a hysteresis cycle within handover decisions so as to prevent unnecessary and unwanted handovers. As an alternative, a precise constraint to handover frequency might be encoded in the VHO algorithm so as to assure a minimum permanence time in a wireless network. This last approach can reduce the algorithm complexity and the overall scalability of the vertical handover algorithm.

However, a deep discussion on the possible techniques to limit the ping-pong effect is out of the scope of this paper.

The detailed descriptions of the behavior of the above vertical handover approaches are discussed in Sections 2.12.5 , respectively.

2.1. RSS-Based VHO Algorithms. The conventional method to initiate a handover (both horizontal and vertical) is by monitoring the mean value of RSS against a predefined acceptable threshold for entry in a candidate network [7]. Since the RSS value suffers from severe fluctuations due to effects of shadowing and fading channels, filtering techniques (i.e., exponential smoothing average [8] and FFT-based methods [9]) should be considered to estimate the trend of RSS signal. In an RSS-based VHO approach, when the measured RSS of the SN drops below a predefined threshold, the RSS of the monitored set of CNs is evaluated in order to select the best network to migrate to. Although the RSS-based VHO method does not aim to optimize communication performance, but only focuses on maintaining a seamless connectivity, it represents the simplest and traditional handover mechanism.

2.2. SINR-Based VHO Algorithms. In the SINR-based approach, Yang et al. [10] compare the received power against the noise and the interference levels in order to obtain a more accurate performance assessment, which brings about a slight increase of computational cost. SINR factor is considered for VHO decisions, as it directly affects the maximum data rate compatible with a given Bit Error Rate (BER). Therefore, when the SINR of the serving network decreases, the data rate and the QoS level decrease too. As a consequence, a SINR-based VHO approach is more suitable to meet QoS requirements, as described in $[10,11]$, and can be used to implement an adaptive data rate procedure. Again, the SINR parameter is also assumed as handover decision metric, combined with traditional RSS factor in order to improve efficient handover executions [11]. RSSbased and SINR-based schemes are both reactive approaches, which means that they aim to compensate for performance degradation when this occurs, that is, whenever either the RSS or the SINR drops below a guard threshold.

2.3. Multiparameter QoS-Based VHO Algorithms. This class of vertical handover takes a connectivity switching decision on the basis of QoS parameters, coming both from the user requirements and the network performance [12, 13]. In [12] a multiparameter QoS-based VHO scheme is described. This technique is representative of a proactive approach based on the regular assessment of the QoS level offered by the current SN, as well as by other CNs. The proposed method attempts to select the best $\mathrm{CN}$ at any time, thus preventing performance degradation and sudden lack of connectivity. It can be based on the simultaneous estimation of a set of parameters - throughput and BER-and in the subsequent evaluation of an objective QoS metric, which is a function of such parameters. Its effectiveness is directly dependent on the ability of the objective QoS metric to mimic subjective Quality-of-Experience of the end-users and on the accuracy of the assessment of the parameters on which the metric is based.

As illustrated in [12], QoS-based VHO is well suited for multimedia applications like real-time video streaming. As a drawback, preventive approaches may lead to high handover frequency and hence lead to algorithmic instability. If numerous unwanted handovers may be executed in order to pursue QoS metric optimization in a proactive fashion, however low reliability in parameter assessment can be the cause of instability even when using a simpler reactive approach. For example, in an RSS-based VHO, the fluctuations of signal strength associated with shadow fading might cause a session to be handed over back and forth between available networks repeatedly. A hysteresis cycle [22], as well as a time constraint on maximum handover frequency (i.e., waiting time parameter [s]) $[8,11,14,18,19]$, represents a solution to prevent an MT to trigger repeatedly undesired handover attempts from one network to another-ping-pong effect.

2.4. Speed-Based VHO Algorithms. The speed-based VHO approach is mainly addressed on Vehicular Ad hoc Networks (VANETs), where users moving at high speeds are hardly able to maintain a service. In this scenario a vehicle-controlled VHO represents the mostly preferred solution, since innovative vehicles, equipped with smart on-board computer, and GPS (Global Positioning System) connectivity, are able to decide whether to make an handover or not [14]. This approach is based on both vehicle speed and handover latency, while in [15] the focus is a jointly improving of three QoS metrics (i.e., delay, jitter, and throughput), while keeping limited the number of vertical handovers. 
In high-mobility environments, either QoS or RSS-based VHO procedure may fail due to the speed and the time that a vehicle is going to effectively spend in a candidate network, compared with the handover latency. It follows that handover algorithms in VANETs should be based on specific factors as vehicle mobility pattern [23] and speed. As a matter, Chen et al. in [23] consider how to reduce both handover delay and packet loss rate by proposing a novel network mobility protocol in VANETs, by exploiting vehicular communications and multihop procedures.

2.5. Location-Based VHO Algorithms. In location-based VHO solutions, the knowledge of location information is exploited to assess the quality of the link between the SN and the MT and to predict its future evolution to some extent on the basis of the MT's estimated path. User's position can be determined in several ways [16], including Time-of-Arrival, Direction-of-Arrival, RSS, and Assisted-GPS techniques.

Examples of location-based VHO algorithms are discussed by Wang et al. in [16], though their proposed technique shows a computational complexity of the handover decision that is rather high, as establishing and updating a lookup table to support a handover margin decision turns out to be time-consuming. In contrast, Kibria et al. [24] develop a predictive framework based on the assumption that the random nature of user mobility implies an uncertainty on his/her future location, which increases with the extension of the prediction interval.

The above descriptions have shown the main vertical handover approaches, mainly based on single metrics (i.e., RSS, SINR, QoS, speed, and location). Still, many handover techniques are based on the combination of two or more handover metrics, which generate most effective VHO decisions, but increase the computational load, since the handover decision exploits a rich set of input criteria. Such techniques are called hybrid (or combined) vertical handover approaches [25]. An example of hybrid approach is given in [11], as described in Section 2.2. Moreover, in [26] Hasswa et al. present a multiparameter VHO decision function, based on monetary cost, power requirements, security parameters, MT's preference, network conditions, and speed. No simulation results are reported for such approach.

Table 1 collects and highlights the main aspects, metrics, and performance parameters of the above discussed VHO approaches, listed in terms of decision metrics (i.e., RSS, SINR, QoS, speed, and location).

This paper proposes a novel vertical handover algorithm-called Power and Location-based Vertical Handover. It is a combined approach, which includes both RSS measurements and a location-based VHO algorithm. In particular, an RSS-VHO approach is used in order to decide on handover execution, while acquiring knowledge on the current environment (i.e., through received power levels, and network channel estimations) [8]. Then, the location information (i.e., the distance from the MT to an access point) is used to estimate QoS parameters (i.e., goodput) and decide for handover executions.
Leveraging such considerations, it follows that our proposed technique can be classified as (i) MCHO, (ii) hybrid approach, and (iii) based on RSS and location information. Its main goals are (i) a maximization of throughput and (ii) a limitation of ping-pong effect.

\section{Proposed Power and Location-Based Vertical Handover (PLB-VHO)}

In this section we shall introduce the Power and Locationbased Vertical Handover (PLB-VHO) technique. In Section 3.1 some basic assumptions are given, regarding the main functionalities of considered dual-mode MTs and APs. A first overview of PLB-VHO approach is presented in Section 3.2, while more detailed descriptions of its two phases, that is, the Distributed Attenuation Map Building (DAMB) and the Enhanced Location-based mode (ELB), are presented in Sections 3.3 and 3.4, respectively.

3.1. Basic Assumption. Without loss of generality, in the illustration of the proposed PLB-VHO scheme, we will consider a dual-mode MT provided with UMTS and WLAN NICs-though the approach we are presenting can be extended to other types of networks - which exploits both RSS and its location measures to assess achievable goodput levels in the WLAN and UMTS networks.

As basic hypothesis, we consider the following assumptions:

(i) the MT moves within an area with double UMTS and WLAN coverage. As it often happens, while UMTS coverage is complete in the considered area, WLAN access is available only in some hot-spot zones;

(ii) the MT is able to determine its location through some auxiliary navigation aid, like GPS, while moving in a visited area;

(iii) both the MT and the APs in the visited network include an application-layer service to calculate a power attenuation map of the visited environment, which can be used to drive handover decisions through a cross-layer approach; (Note that for the sake of simplicity, we will use the generic term access point to indicate a network node providing access to the wireless network for both the WLAN, and the UMTS technologies.)

(iv) the AP includes a server application, while the MTs attached to it a client application. Client applications regularly deliver to the server application RSS samples linked to location information. The server application calculates an attenuation map from the received samples and broadcasts it to all MTs attached to the network.

3.2. PLB-VHO Overall Operation. The PLB-VHO scheme includes two procedures, that is, (i) a Distributed Attenuation Map Building (DAMB) and (ii) an Enhanced Location-based Vertical Handover (ELB-VHO), exploiting the calculated attenuation maps for the WLAN and the UMTS networks. 
TABLE 1: Classification of handover algorithms on the basis of decision metrics and performance results.

\begin{tabular}{|c|c|c|c|}
\hline VHO Decision Metric & VHO algorithm & Main aspects & Performance \\
\hline \multirow{3}{*}{ RSS } & $\begin{array}{l}\text { Ayyappan and } \\
\text { Dananjayan [7] }\end{array}$ & $\begin{array}{l}\text { This technique considers both hard and soft MCHO version } \\
\text { between WLAN and CDMA networks. The RSS parameter is } \\
\text { monitored against unwanted power level fluctuations. }\end{array}$ & Throughput \\
\hline & Inzerilli and Vegni [8] & $\begin{array}{l}\text { This approach is an MCHO technique between WLAN and } \\
\text { CDMA networks. It focuses on the minimization of } \\
\text { ping-pong effect by channel estimation techniques and } \\
\text { waiting time parameter. }\end{array}$ & $\begin{array}{l}\text { Throughput and } \\
\text { handover frequency }\end{array}$ \\
\hline & Xie and $\mathrm{Wu}[9]$ & $\begin{array}{l}\text { The algorithm makes handoff decisions after analyzing the } \\
\text { signal strength fluctuation caused by slow fading through } \\
\text { FFT. The method strongly reduces the number of handovers. } \\
\text { It can be applied to both horizontal and vertical handovers. }\end{array}$ & $\begin{array}{l}\text { Handover frequency } \\
\text { and delay }\end{array}$ \\
\hline \multirow[t]{2}{*}{ SINR } & Yang et al. [10] & $\begin{array}{l}\text { This technique results in a QoS oriented VHO, since the } \\
\text { SINR factor is strictly affecting the QoS level. }\end{array}$ & Throughput \\
\hline & Vegni et al. [11] & $\begin{array}{l}\text { It is an MCHO technique between WLAN and CDMA } \\
\text { networks. The goal is to limit the ping-pong effect by } \\
\text { preventive vertical handovers. Since the SINR factor is } \\
\text { combined with RSS parameter, the technique results in } \\
\text { combined-metric based VHO approach for preventing } \\
\text { unnecessary VHOs. }\end{array}$ & $\begin{array}{l}\text { Throughput and } \\
\text { handover frequency }\end{array}$ \\
\hline
\end{tabular}
unnecessary VHOs.

In this technique the QoS parameters are both subjective and

$\begin{array}{ll} & \text { Vegni et al. [12] } \\ \text { QoS } & \text { VHO technique between WLAN and UMTS networks, in an } \\ & \text { IEEE } 802.21 \text { network architecture. }\end{array}$

Jesus et al. [13]

Vegni and Esposito [14]

Speed

Esposito et al. [15]

Chen et al. [23]

Wang et al. [16]

Location

Kibria et al. [24]

Inzerilli et al. [18]
The handover technique is QoS-oriented and is based on context-aware information coming from both the network and the user. The handover decision is negotiated by both the MT and the network (i.e., UMTS and WLAN) in an IEEE 802.21 * network architecture.

This technique is a vehicle-controlled VHO, based on speed and handover delay. It addresses on VANETs.

Seamless connectivity

Maximum allowed number of active users

Throughput and handover frequency

Throughput, jitter, delay and handover frequency improving QoS metrics.

This is a novel network mobility management protocol for VANETs, by exploiting traditional vehicle-to-vehicle communications.

This technique is limited on a horizontal handover approach, for both hard and soft versions. The MT's location is exploited for adaptive handovers.

Handoff delay and packet loss rate

Dropped calls and outage probability

Hysteresis margins

It is an MT controlled VHO technique, based on MT's location information to predict handovers.

This method is driven by MT's location in order to prevent vertical handovers between WLAN and UMTS networks. The limitation of ping-pong effect is acted by the use of the waiting time parameter.
Throughput and number of vertical handovers

\footnotetext{
* The IEEE 802.21 standard provides quick handovers of data sessions across heterogeneous networks with small switching delays and minimized latency [27]. The handover procedures could become more flexible and appropriate with this standard, by exploiting the Media Independent Handover (MIH) functional model. The IEEE 802.21 focuses on protocol aspects rather than handover algorithms. In this table the IEEE 802.21 is not investigated since it is out of the scope of this paper.
} 
Namely, an MT switching on in the visited environment is initially unaware of the signal power spatial distribution (i.e., no knowledge of access point's location and associated power levels is available) for the available UMTS and WLAN networks. The MT will enter the DAMB-mode and interrogate the serving APs for each network being visited, in order to obtain the attenuation maps. The attenuation maps can be returned immediately if available in the AP, as a result of the interaction of the AP with other MTs previously roaming in the network and collaborating to the Attenuation Map Building.

Once provided with both the attenuation maps from both the WLAN and UMTS networks, the MT can select the best network and enters the Enhanced Location-based (ELB) mode. While roaming it will then exploit the received attenuation maps along with the current location information to assess achievable goodput in both the UMTS and WLAN networks and take handover decisions to optimize goodput. Namely, knowledge of the expected spatial distribution of the power attenuation in the surrounding area of the current location allows taking more accurate and stable estimates of the achievable goodput. The handover algorithm used in this phase is then called as Power and Location-based Vertical Handover (PLB-VHO).

In the ELB-mode the MT will continue collecting RSS samples and deliver them to the serving AP, which will contribute to the update of the attenuation maps in the serving access points. When the MT moves towards new unvisited zones, requiring for instance change of serving access point in the same network, new attenuation maps might have to be built and hence the MT re-enters the DAMB-mode. It will transit to the ELB-mode again once the updated attenuation maps have been created.

Figure 1 depicts the essentials of the PLB-VHO, highlighting the two modes just described. In the DAMBmode the MT exploits an interrogating-phase with the nearest access point. In the ELB-mode the MT relies on a more elaborate $\mathrm{VHO}$ algorithm, while it keeps refining its knowledge of the visited environment (Attenuation Map Update).

Figure 2 shows an example scenario with a mesh network of five MTs (i.e., MT1, MT2, MT3, MT4, MT5) with dual WLAN and UMTS NICs, two WLAN access points (i.e., AP1 WLAN, AP2 WLAN), and one UMTS access point (i.e., AP1 UMTS). The five MTs roam in an area covered by the two WLAN APs and a single UMTS AP. Namely, MT2, MT3, MT5 roam in the AP1 WLAN area, while MT1 and MT4 roam in the AP2 WLAN area.

In Figure 2 we use dotted lines for time scales in the intervals of DAMB-mode, while continuous lines are used in the intervals of ELB mode. The picture shows only the messages of map requests from the MTs and map replies by the APs, while delivery of RSS samples from MTs to APs are neglected. The dynamics of message exchange are the following:

(i) MT1, MT2, and MT3 have already issued a map request to AP1 WLAN, AP2 WLAN, and AP1 UMTS prior time $t=0$;

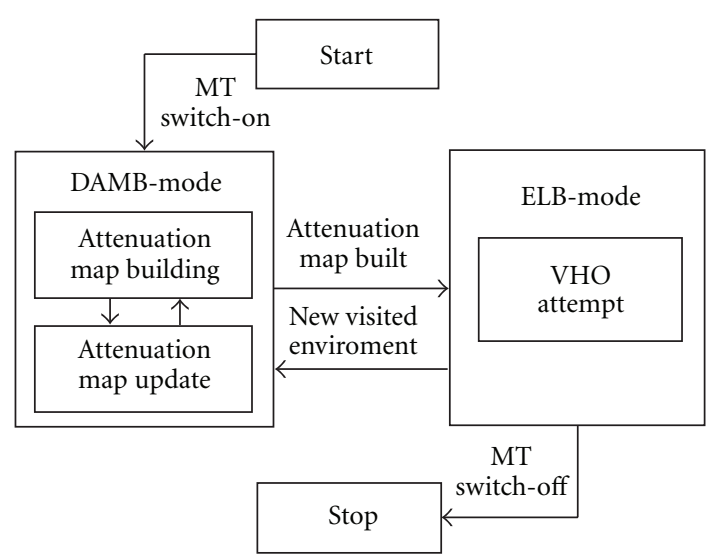

FIgURE 1: Finite state machine for the PLB-VHO algorithm.

(ii) at time $t=0$ an attenuation map becomes available at AP1 UMTS and it is issued to MT1, MT2 and MT3;

(iii) MT2 and MT3 will instead wait for time $t=9$ to receive a map from AP1 WLAN, while MT1 will wait for time $t=12$ for the map;

(iv) MT4 and MT5 enter the area following $t=0$ and hence receive the attenuation map from AP1 UMTS immediately, after their request. On the contrary, reception of WLAN maps for MT4 and MT5 occurs in times $t=9$ and $t=12$, respectively, that is, as soon as they become available.

3.3. Distributed Attenuation Map Building (DAMB-Mode). From mobile switch-off up to the completion of both UMTS and WLAN Attenuation Map Building, the mobile terminal uses the DAMB procedure to obtain attenuation maps and works jointly with the other neighboring mobile terminals.

During the DAMB-mode each MT selects and interrogates the nearest access points of the UMTS and WLAN networks, collects a set of RSS samples for the Attenuation Map Building process, and delivers them to the selected access points. Availability of a power attenuation map derived from RSS measures makes it possible to apply a more sophisticated method for handover management and optimization of goodput without waiting for severe performance degradation prior handover initiation.

The spatial distribution of the power attenuation associated to the monitored UMTS and WLAN access points are calculated by each AP simply as the difference between the access point transmission power and the RSS samples received by the MTs connected to the AP, that is, by taking the difference between the nominal transmitted power and the short-term time average of the RSS. Averaging is required in order to smooth fast fluctuations produced by multipath signal reflections and can be performed by means of a mean filter applied to the attenuation sample series multiplied by a sliding temporal window.

Let us assume that an access point governs an area partitioned into a lattice of $\widetilde{M}_{H} \times \widetilde{M}_{V}$ square zones, each of them with a width $w_{\text {zone }}$. In general, this parameter is 


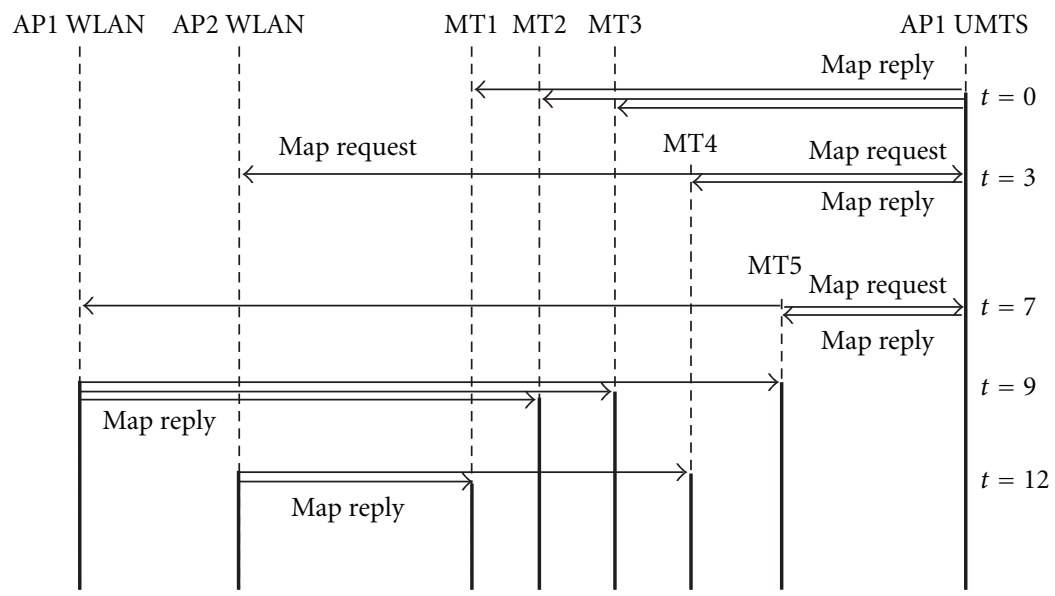

FIGURE 2: DAMB procedure in a mesh network example scenario.

different for UMTS and WLAN networks, in accordance to the maximum rate of change of the received power signals. While moving in that area, an MT measures the attenuation in each visited zone, associates it with its current location, and delivers it to the serving access point.

Let $n$ be the discrete time index, and let $a_{j}[n]$ be the attenuation measured in the $j$ th zone at time $n$. Then, the Moving Average (MA) attenuation estimation (i.e., $A_{j}^{\mathrm{MA}}[n]$ ) on a sliding window of length $K$ is

$$
A_{j}^{\mathrm{MA}}[n]=\frac{1}{K} \sum_{i=n-K+1}^{n} a_{j}[i], \quad n \geq K .
$$

Averaging over the last $K$ samples allows reducing the impact of instantaneous power fluctuations in attenuation detection and reduces the power error estimation. On the other hand, as the mobile terminal is assumed to be moving, the length of the moving window cannot be too large. As an alternative, an Exponential Smoothing Average filter with time constant $t$ can be applied, so that

$$
A_{j}^{\mathrm{ESA}}[n]=\alpha \cdot A_{j}[n-1]+(1-\alpha) \cdot a_{j}[n],
$$

where $\alpha=\exp \left(-\left(t_{n}-t_{n-1}\right) / \tau\right)$.

Although (1) and (2) have the same computational cost and similar performance, (2) requires smaller quantity of memory to store the measured time series $\left\{a_{j}[n]\right\}$. Furthermore, since Moving Average filters are prone to outliers, a more robust estimate can be computed by replacing the linear mean filter with a (nonlinear) median filter.

When each zone of the lattice has been visited at least once by an MT, the attenuation map is completed. However, it is possible that a complete visit of all the zones of the map can take a long time, and perhaps it never accomplishes. As a consequence, in order to speed up the Attenuation Map Building process it is possible to resort to interpolation in order to assign an attenuation value to locations that have not yet been visited.

Namely, let us assume that the $j$ th zone, with center in $\left(x_{j}, y_{j}\right)$, has not been assigned a power value yet, and let $j_{1}$, $j_{2}$ and $j_{3}$ be the nearest three locations whose attenuation has already been measured. We can estimate the attenuation $A_{j}$ of zone $j$ by applying linear algebra and using the equation of a plane passing through three points

$$
\operatorname{det}\left(\begin{array}{lll}
x_{j}-x_{1} & y_{j}-y_{1} & A_{j}-A_{1} \\
x_{2}-x_{1} & y_{2}-y_{1} & A_{2}-A_{1} \\
x_{3}-x_{1} & y_{3}-y_{1} & A_{3}-A_{1}
\end{array}\right)=0
$$

Through simple manipulation of (3), we can easily obtain a direct formula for interpolation of $A_{j}$, such as

$$
\begin{aligned}
A_{j}= & \frac{A_{2}\left(x_{3} y_{1}-x_{j} y_{1}-x_{1} y_{3}+x_{j} y_{3}+x_{1} y_{j}-x_{3} y_{j}\right)}{x_{3}\left(y_{1}-y_{2}\right)+x_{1}\left(y_{2}-y_{3}\right)+x_{2}\left(-y_{1}+y_{3}\right)} \\
& +\frac{A_{1}\left(-x_{3} y_{2}+x_{j} y_{2}+x_{2} y_{3}-x_{j} y_{3}-x_{2} y_{j}+x_{3} y_{j}\right)}{x_{3}\left(y_{1}-y_{2}\right)+x_{1}\left(y_{2}-y_{3}\right)+x_{2}\left(-y_{1}+y_{3}\right)} \\
& +\frac{A_{3}\left(x_{j} y_{1}+x_{1} y_{2}-x_{j} y_{2}-x_{1} y_{j}+x_{2}\left(-y_{1}+y_{j}\right)\right)}{x_{3}\left(y_{1}-y_{2}\right)+x_{1}\left(y_{2}-y_{3}\right)+x_{2}\left(-y_{1}+y_{3}\right)} .
\end{aligned}
$$

It is worth highlighting that linear interpolation through (3) brings some errors in the attenuation map. In general, a sufficient number of visited zones have to be achieved prior completion of the attenuation map. Such a number is also dependent on the actual path of the MT in the lattice.

Let VZ $[n]$ be the set of visited zones by an MT up to time $n$ at time $n$. Then, in order to evaluate the degree of reliability of the attenuation map at time $n$, we employ a Map Reliability Index $(M R I)$ at time $n$, defined as follows:

$$
\operatorname{MRI}[n]=\frac{\|V Z[n]\|}{\widetilde{M}_{H} \cdot \widetilde{M}_{V}},
$$

where $\widetilde{M}_{H}$ and $\widetilde{M}_{V}$ represent the number of horizontal and vertical zones in the neighborhood, respectively.

We can empirically set a threshold value $\mathrm{MRI}^{\mathrm{TH}}$ for the index in (5) beyond which the knowledge of the visited environment is regarded as acceptable. Only when this threshold 
is exceeded, interpolation is applied. Thus, the attenuation map will be filled in partially with measured attenuations and partially through linear interpolation, respectively.

Even after Attenuation Map Completion, when the MT enters the ELB-mode (see Figure 1), power samples continue being collected and used as in (2) in order to increase the accuracy of each map. Conversely, when for the current location the MRI falls below MRI ${ }^{\mathrm{TH}}$ a transition from the $E L B$-mode to the DAMB-mode is performed (see Figure 1).

3.4. Enhanced Location-Based (ELB) Mode. A more significant parameter than measured RSS for comparing performance of two wireless links is the expected goodput, that is, the net transmission throughput out of the percentage of service outage.

In general, the goodput experienced by an MT in a wireless cell depends on the bandwidth allocated to the MT for the requested services and on the channel quality. When inelastic traffic (e.g., real-time flows over UDP) is conveyed the goodput (i.e., GP [Mbps]) can be approximated to the net traffic received out of channel errors and given by

$$
\mathrm{GP}=\mathrm{BW} \cdot\left(1-P_{\text {out }}\right),
$$

where BW [bps] is the bandwidth allocated to the MT and $P_{\text {out }}$ is the service outage probability in the considered channel. Instead, when elastic traffic is conveyed (typically when TCP is used for data downloading/uploading), throughput tends to decrease with increasing values of $P_{\text {out }}$ as an effect of the TCP congestion control algorithm.

The parameter BW is in general a function of the wireless link nominal capacity and is conditioned by the Medium Access Control algorithm that is used and sometimes of the experienced $P_{\text {out }}$, for example, in those technologies using adaptive modulation. In the UMTS network $P_{\text {out }}$ can be theoretically calculated [28], using the following formula:

$$
P_{\mathrm{out}}^{\mathrm{UMTS}}=\operatorname{Pr}\left\{\frac{E_{b, \mathrm{Tx}}^{\mathrm{UMTS}}}{\sigma_{N_{\mathrm{UMTS}}}^{2}+\gamma I_{0_{\mathrm{UMTS}}}} \cdot \frac{1}{A^{\mathrm{UMTS}}\left(d_{\mathrm{UMTS}}\right)} \leq \mu_{\mathrm{UMTS}}\right\},
$$

where $E_{b, \mathrm{Tx}}^{\mathrm{UMTS}}$ is the transmitted bit energy, $\mu$ and $\gamma$ are parameters dependent on the signal and interference statistics, respectively, and $\sigma_{N}^{2}$ is the receiver noise power. $I_{0}$ is the inter- and intracell interference power, and can be calculated in terms of the number of effective interfering users (i.e., $N_{\text {interf }}$ ) as follows:

$$
I_{0}=\frac{N_{\text {interf }}}{G_{\text {spread }}} E_{b, \text { Tx }}^{\text {UMTS }}
$$

where $G_{\text {spread }}$ is the WCDMA spreading factor. Finally, the parameter $A^{\text {UMTS }}$ ( $d_{\text {UMTS }}$ ) is the overall power loss, expressed as

$$
A^{\mathrm{UMTS}}\left(d_{\mathrm{UMTS}}\right)=\frac{P_{\mathrm{Tx}}}{P_{\mathrm{Rx}}}=\left(\frac{4 \pi}{\lambda}\right)^{2} \frac{d_{\mathrm{UMTS}}^{\beta}}{G_{\mathrm{Tx}}^{\mathrm{Ant}} G_{\mathrm{Rx}}^{\text {Ant }}},
$$

which depends on the MT's distance $d_{\text {UMTS }}$ from the UMTS base station.
Let us initially assume for simplicity that all wireless cells have an isotropic behavior [20]. Expected goodput is then calculated as a function of a single variable, that is, the MT's distance from the access point of the network cell the mobile device is visiting.

The service outage probability for a WLAN network $P_{\text {out }}^{\text {WLAN }}$ can be theoretically calculated in a similar fashion to (7), using the following formula:

$$
P_{\mathrm{out}}^{\mathrm{WLAN}}=\operatorname{Pr}\left\{\frac{E_{b, \mathrm{Tx}}^{\mathrm{WLAN}}}{\sigma_{N_{\mathrm{WLAN}}}^{2}} \cdot \frac{1}{A^{\mathrm{WLAN}}\left(d_{\mathrm{WLAN}}\right)} \leq \mu_{\mathrm{WLAN}}\right\} .
$$

We remark that, with respect to the UMTS W-CDMA case, cochannel interference effects are not present due to the different structure of the physical layer. For compactness of notation we observe that (10) can be formally seen as a special case of (7) obtained for $\gamma=0$.

Let us define as the range of an isotropic cell the distance $R_{\text {cell }}$ from the cell centre beyond which the outage probability exceeds the maximum acceptable value $\widetilde{P}_{\text {out }} . R_{\text {cell }}$ can be obtained resolving the above equations or empirically, through measurement on the network. As an alternative, typical value for well-known technologies can be used, for example, $[29,30]$.

Let $\tilde{\mu}$ be the threshold corresponding to signal to a given QoS level based on (7) for UMTS networks and on (10) for WLAN cells, respectively. Then, as the path loss $A_{d}(d)$ for a link of length $d$ is approximately proportional to $d^{\beta}$, the received Signal-to-Noise and Interference ratio (i.e., $\operatorname{SINR}(d)$ ) can be written as (see [31])

$$
\operatorname{SINR}(d)=\tilde{\mu}\left(\frac{R_{\text {cell }}}{d}\right)^{\beta}
$$

For a given location at distance $d^{\text {WLAN }}<R_{\text {cell }}^{\text {WLAN }}$ from a WLAN access point, and at distance $d^{\text {UMTS }}<R_{\text {cell }}^{\text {UMTS }}$ from an UMTS base station, since in free space $\beta=2$, while in more complex environments $\beta>2$, the goodput $\mathrm{GP}(d)$ at distance $d$ from the access point can be computed as a derivation from (6), with the following approximated formula:

$$
\begin{array}{r}
\mathrm{GP}^{(k)}\left(d_{(k)}\right)=\mathrm{BW}_{\text {max }}^{(k)} \cdot \operatorname{Pr}\left\{d_{(k)}<R_{\text {cell }}^{(k)}\right\}, \\
k \in\{\text { UMTS, WLAN }\}
\end{array}
$$

whose value is set to zero if the distance is greater than the cell range, while $\mathrm{GP}^{(k)}\left(d_{(k)}\right)=\mathrm{BW}_{\text {max }}^{(k)}$, when the mobile terminal is lying at the center of the wireless cell.

Handover can be initiated when the estimated goodput of the Serving Network ( $\mathrm{SN}$ ) is lower than that of the Candidate Network (CN). Namely, in the case of vertical handover from WLAN to UMTS, the following condition holds:

$$
\mathrm{GP}_{\max }^{\mathrm{UMTS}}\left(d_{\mathrm{UMTS}}\right)<G \mathrm{P}_{\max }^{\mathrm{WLAN}}\left(d_{\mathrm{WLAN}}\right) .
$$

It is worth noticing that when handovers are too frequent, the quality as perceived by the end-user can significantly degrade in addition to the waste of battery charge. Then it can be useful to limit handover frequency by imposing a minimum time interval between two consecutive 

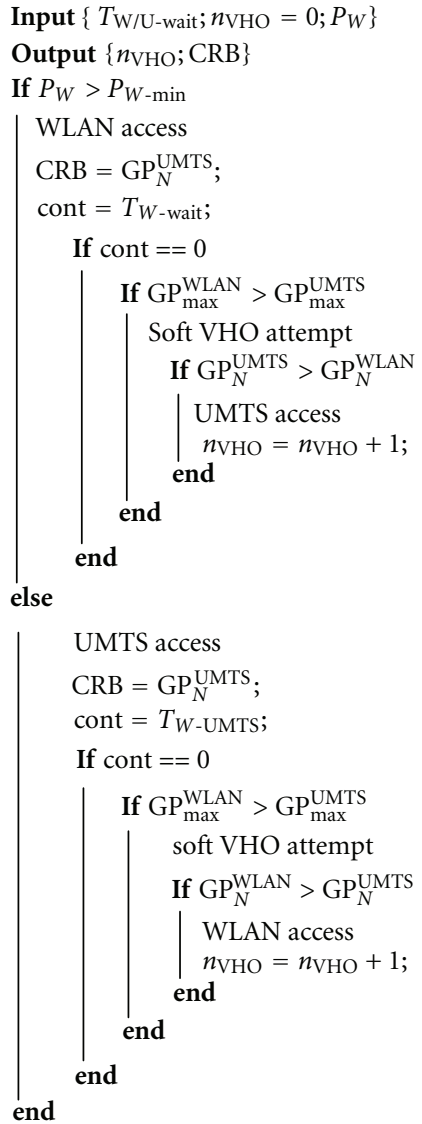

Figure 3: Pseudo-code for vertical handover algorithm in ELBmode.

handovers (i.e., applying the waiting time constraint [s]), possibly different for the cases of UMTS-to-WLAN handover and WLAN-to-UMTS handover, as in $[8,14,15,18]$. As an alternative, also a hysteresis cycle in handover initiation process can be introduced. We remind from [15] that the waiting time parameter has been defined as an interswitch time period, during which the handover process enters an idle mode. For instance, if a mobile terminal moves at $0.5 \mathrm{~m} / \mathrm{s}$, a 10-second waiting time results in 50 meters covered by the user, before the handover process is reactivated. This approach results are necessary to avoid a high handover frequency.

Figure 3 depicts the pseudocode for the Enhanced Location-based (ELB) process, which uses (13) to drive handover decisions and exploits a waiting time, different for WLAN and UMTS (i.e., $T_{\mathrm{W} / \mathrm{U} \text {-wait }}[\mathrm{s}]$ for WLAN, and UMTS, resp.) between consecutive handovers to limit the number of executed handovers (i.e., handover frequency).

In the location-based approach presented so far the goodput is estimated simply on the basis of the distance $d$ from the center of a wireless cell. This method is applicable when the coordinates of the center of the cells and the cell range are known a priori. In addition, this goodput model assumes an isotropic access point source and no obstacles between the MT and the access point.

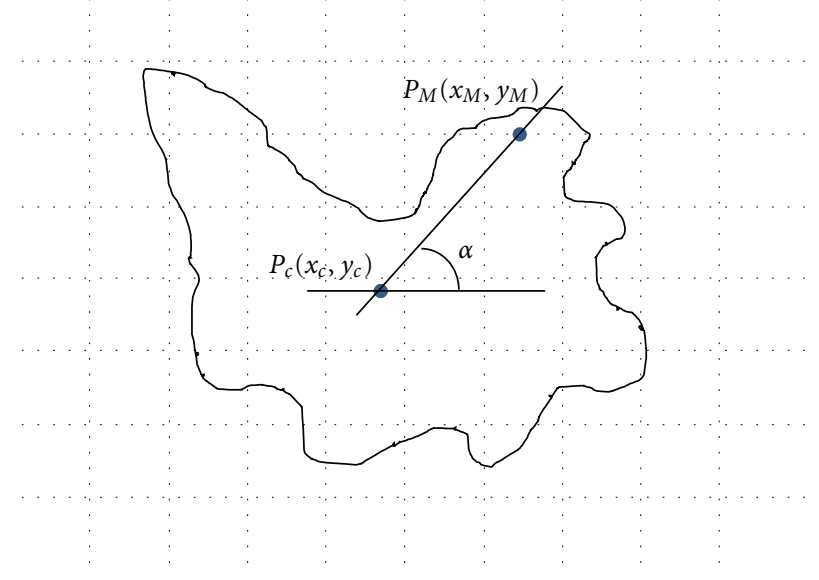

FIgURE 4: Anisotropic cell model.

In this section we will exploit the Distributed Attenuation Map Building phase in order to derive a more realistic estimate of the goodput by relaxing the hypothesis of isotropic cells.

In order to exploit the PLB-VHO approach, it is first necessary to obtain (i) a goodput estimation approach adapted for anisotropic cells, and (ii) a method to derive wireless cell geometry from the Distributed Attenuation Map Building.

We assume a generic-shape cell model and estimate goodput as a function of the MT's line of sight direction $\alpha$, (i.e., the direction of the line drawn from the access point's location $P_{c}=\left(x_{c}, y_{c}\right)$ to the MT's current position $P_{M}=$ $\left(x_{M}, y_{M}\right)$, as shown in Figure 4$)$. Namely, $\alpha$ is calculated as follows:

$$
\alpha=\arctan \left(\frac{y_{M}-y_{c}}{x_{M}-x_{c}}\right) .
$$

For a cell with access point placed in $P_{c}$, we define the radius of the cell $R_{\text {cell }}$ as a function of the line of sight $\alpha$, which represents the distance $R_{\text {cell }}=R_{\text {cell }}\left(\alpha ; P_{c}\right)$ from the cell centre along the line of sight $\alpha$ beyond which the outage probability exceeds the maximum acceptable value $\widetilde{P}_{\text {out }}$.

Hence, the goodput $\mathrm{GP}^{(k)}\left(d_{(k)}, \alpha_{(k)}\right)$ at distance $d_{(k)}$ along the line of sight $\alpha$ can be calculated for each zone with the following approximated formula, which replaces (12) for the case of anisotropic cells:

$$
\mathrm{GP}^{(k)}\left(d_{(k)}, \alpha_{(k)}\right)=\mathrm{BW}_{\max }^{(k)} \cdot \operatorname{Pr}\left\{d_{(k)}<R_{\text {cell }}^{(k)}\left(\alpha ; P_{c}\right)\right\},
$$

where, as in (12), $k$ is the index denoting the corresponding wireless network, that is, $k \in\{$ UMTS, WLAN $\}$. Handover decisions are still taken on the basis of (13).

We can calculate the function $R_{\text {cell }}^{(k)}\left(\alpha ; P_{c}\right)$ by using the Attenuation Map. In fact, for a given direction $\alpha$, we can consider the set of zones lying along the corresponding line of sight. Using (3) the attenuation profile along the line of sight can be easily computed. Then, the cell range along direction $\alpha$ can be set to the distance for which the attenuation equals to the maximum attenuation $A_{\text {max }}$, beyond which the outage probability exceeds the maximum acceptable value $\widetilde{P}_{\text {out }}$. 
In addition, in zone $Z_{j}$ with center in $\left(x_{j}, y_{j}\right)$ characterized by an attenuation $A_{j}^{(k)}$ the average goodput can be evaluated as

$$
\mathrm{GP}^{(k)}\left(d_{(k)}, \alpha_{(k)}\right)=\mathrm{BW}_{\max }^{(k)} \cdot \operatorname{Pr}\left\{A_{j}^{(k)}<A_{\max }^{(k)}\right\}
$$

where $\operatorname{Pr}\left\{A_{j}^{(k)}<A_{\max }^{(k)}\right\}$ is obtained as an analogy from $\operatorname{Pr}\left\{d_{(k)}<R_{\text {cell }}^{(k)}\left(\alpha ; P_{c}\right)\right\}$.

\section{Performance Evaluation and Comparisons}

Simulation results for PLB-VHO technique are now presented and compared with other vertical handover schemes. Namely, a multiparameter vertical handover-that is, DRIVHO, Data Rate, and Interference-based Vertical Handover, [11] — is considered along with the two single-parameter vertical handover approaches, such as (i) a traditional PowerBased Vertical Handover (PB-VHO) [8] and (ii) a simple Location-Based Vertical Handover (LB-VHO) [18], from which PLB-VHO is derived. Basically, simulated trends for all four algorithms - PB, LB, DRI, and PLB_-represent different realistic cases in a dual-mode WLAN/UMTS MT using in turn one of the four algorithms.

As described in [8], PB-VHO uses power measurements in order to initiate VHOs, while LB-VHO employs mobile location information to optimize MT's goodput [18]. PLB$\mathrm{VHO}$ integrates then power estimations and location information in order to enhance the use of location information and apply it to anisotropic cells. Finally, DRI-VHO-also referred to as C-VHDF (Combined-Vertical Handover Decision Function) in [11] - is a VHO hybrid approach, whose purpose is goodput optimization, as well as in PLB and $\mathrm{PB}$. In DRI-VHO, RSS measurement is used to drive VHO in a first phase (i.e., handover initiation), while data rate estimation from SINR guides the handover accomplishment phase (i.e., handover execution). The DRI-VHO aims to maximize throughput, through the optimization of Data Rate gain parameter [Bps], defined as the increment of data rate resulting from the execution of a vertical handover. More details of DRI-VHO technique are given in [11].

In the scenarios simulated using Matlab 7.6, an MT moves in a heterogeneous network grid with 3 UMTS and 20 WLAN cells. One hundred network scenarios have been generated, where the location of the WLAN access points and UMTS base stations are randomly varied. In each scenario the MT moves with a constant speed (i.e., $0.5 \mathrm{~m} / \mathrm{s}$, corresponding to a pedestrian speed) along a random path inside the heterogeneous grid for a simulated time around 1 hour and 20 minutes.

The Okomura-Hata model for the signal power attenuation [32] has been employed together with an AWG (Additive White Gaussian) channel model. In addition, the following parameter set has been employed, as well as in $[8,18]$ : (i) the transmitted power in the middle of UMTS cells equal to $43 \mathrm{dBm}$ and (ii) UMTS/WLAN receiver sensitivities $P_{\mathrm{U} / \mathrm{W}-\mathrm{min}}$ and $P_{\mathrm{U} / \mathrm{W}-\mathrm{TH}}$ threshold equal to $-100 \mathrm{dBm}$.

During the walk, the MT moves in an area totally covered by UMTS, which means that UMTS coverage is always guaranteed, though with different levels of throughput. Occasionally the MT enters some WLAN cells, which are hotspots where the MT can reach higher levels of throughput.

In Figure 5(a) performance of the four algorithms is evaluated in terms of the number of the executed vertical handovers versus the waiting time parameter, as introduced in Section 3.4. As expected, the number of VHOs obtained with the PB-VHO is significantly lower than that for all the other techniques (i.e., LB-VHO, PLB-VHO, and also DRI-VHO) whose curves are roughly overlapping, except for that relative to $\mathrm{LB}-\mathrm{VHO}$, which is significantly higher, when the waiting time is set to 0 . This means that performance optimization pursued by the LB, PLB, and DRI is achieved at the expenses of an increase handover frequency, though PLBVHO results as more effective than DRI-VHO and LB-VHO, in terms of a decreasing average of handover occurrences, independently on the specific values considered for the waiting time parameter. In contrast, the worse performance is given by LB-VHO particularly for low values of the waiting time. This is probably due to the fact that the location-based handover decision scheme is less precise in LB-VHO than in PLB-VHO as it does not consider the actual shape of the wireless cells and approximates them to simple circles.

Table 2 shows, in more detail, the collected statistics of the number of VHOs experienced with the PB, LB, DRI, and PLB approaches, respectively. For each of them two columns are given. In the first column the mean values of the number of $\mathrm{VHO}$, also depicted in Figure 5(a), are reported for different values of waiting time (i.e., from 0 up to $120 \mathrm{~s}$ ). These are calculated by averaging the total number of $\mathrm{VHO}$ values registered at the end of each simulation over the considered 100 scenarios for each value of the waiting time. In the second column the so-called Dispersion Index (i.e., defined as the ratio between the standard deviation and the mean value over the considered 100 scenarios) is reported for different values of waiting time (i.e., from 0 up to $120 \mathrm{~s}$ ). (The Dispersion Index represents the relative variation of the collected samples with respect to the mean value.) It is observed that the number of vertical handovers with DRI-VHO is on average two times greater-equal to $104 \%$ increment-than that obtained with $\mathrm{PB}-\mathrm{VHO}$, while DRI-VHO increases the handover occurrences with respect to PLB-VHO (i.e., on average $21 \%$ increment). In contrast, DRI-VHO shows on average a low number of handovers-equal to $11 \%$ decrement-with respect to LBVHO approach, while PLB-VHO has a higher number of vertical handovers-equal to $68 \%$ increment-than that for PB-VHO. Finally, PLB-VHO results on average in $26 \%$ reduction of the number of vertical handovers, with respect to the LB-VHO approach.

In order to evaluate the effectiveness of a vertical handover technique along with the number of VHOs, the total number of data, that is, Cumulative Received Bits (CRBs), is also considered. Such statistics has to be regarded as more important in the evaluation the VHO performance, as the number of VHOs, when these are an issue, can be explicitly limited by setting the waiting time parameter properly. 


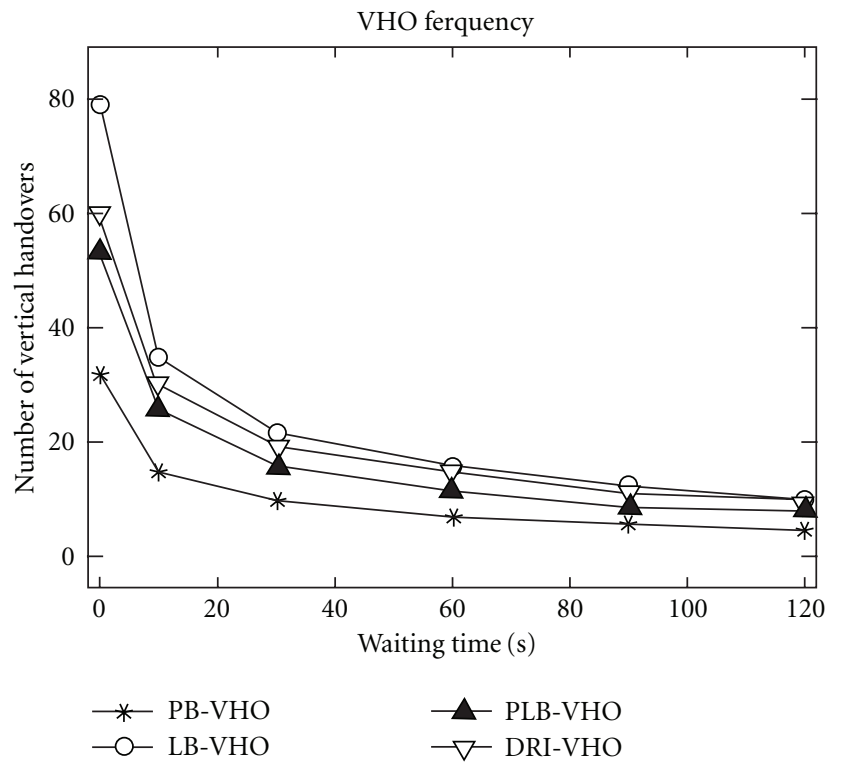

(a)

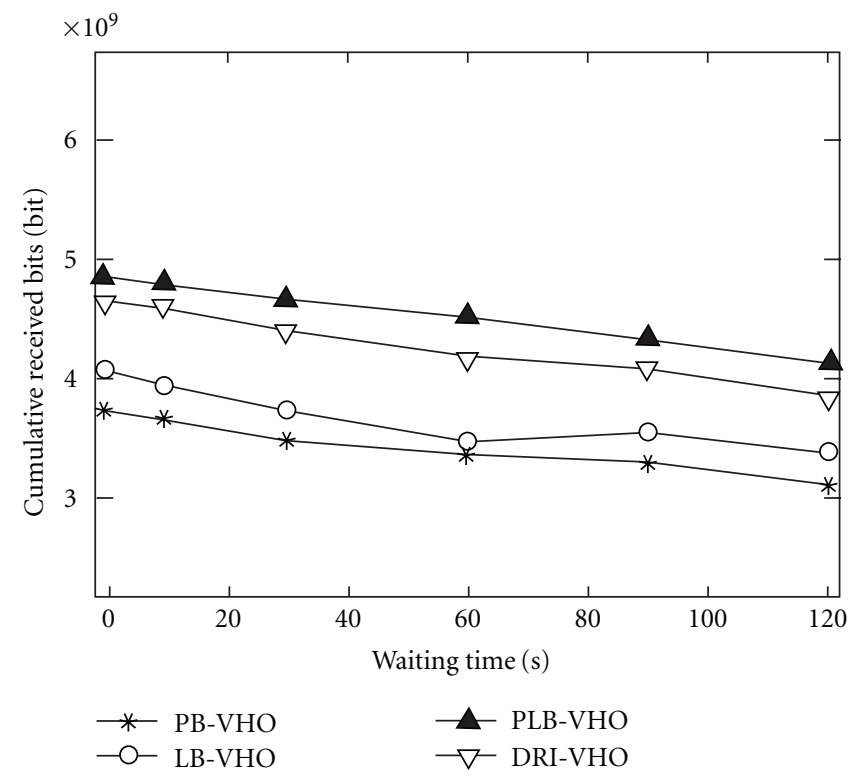

(b)

FIGURE 5: (a) Average of number of vertical handover occurrences for PB, LB, DRI, and PLB. The handovers are performed by a MT during its path, for different values of waiting time constraints. Performances have been obtained over 100 simulation scenarios. (b) Average of CRBs performance for PB, LB, DRI, and PLB-VHO versus different values of waiting time parameter. Performances have been obtained over 100 simulation scenarios.

TABLE 2: Statistics for the number of VHO occurrences for PB, LB, DRI-VHO, and PLB-VHO approaches.

\begin{tabular}{lcccccccc}
\hline $\begin{array}{l}\text { Waiting time } \\
{[\mathrm{s}]}\end{array}$ & PB mean & $\begin{array}{c}\text { PB disp. } \\
\text { index }\end{array}$ & LB mean & $\begin{array}{c}\text { LB disp. } \\
\text { index }\end{array}$ & DRI mean & $\begin{array}{c}\text { DRI disp. } \\
\text { index }\end{array}$ & $\begin{array}{c}\text { PLB mean } \\
\text { index }\end{array}$ \\
\hline 0 & 31.8 & $81.58 \%$ & 79.2 & $24.29 \%$ & 60.1 & $36.98 \%$ & 53.1 & $51.28 \%$ \\
10 & 14.6 & $65.23 \%$ & 34.6 & $25.42 \%$ & 30.2 & $40.39 \%$ & 25.8 & $50.29 \%$ \\
30 & 9.6 & $62.73 \%$ & 21.4 & $31.16 \%$ & 19.2 & $34.09 \%$ & 16 & $42.08 \%$ \\
60 & 6.8 & $68.20 \%$ & 15.8 & $49.72 \%$ & 15.1 & $34.54 \%$ & 11.6 & $44.36 \%$ \\
90 & 5.6 & $64.76 \%$ & 12.3 & $49.97 \%$ & 11.2 & $36.88 \%$ & 8.8 & $37.42 \%$ \\
120 & 4.6 & $76.82 \%$ & 10 & $55.17 \%$ & 9.4 & $24.67 \%$ & 8 & $39.08 \%$ \\
\hline
\end{tabular}

In Figure 5(b) the CRBs versus the waiting time parameter is displayed. The PLB-VHO has visibly the best performance of all the four approaches. Namely, PB-VHO does not aim at goodput performance optimization but it rather limits computational cost and simply aims to recover from connectivity loss. LB-VHO performs a rough estimation of the achievable goodput assuming circular cells, unlike PLBVHO which estimates cell shapes more accurately. Finally DRI-VHO, which aims at goodput optimization on the basis of SINR estimation, turns out to be less accurate in handover decision than PLB-VHO, still as it does not exploit location information. This is evident from the fact that DRI-VHO experiences both a higher number of handovers and results into lower values of goodput.

Table 3 provides the statistics for the CRBs of the four considered algorithms. As in Table 2, two columns for each algorithm are provided. Analogously, The first column reports the mean value of the CRBs parameter versus the waiting time parameter, while the second column reports the relevant Dispersion Index versus the waiting time parameter. The CRBs mean values for PLB-VHO and DRI-VHO at 0 second waiting time are around $4.90 \mathrm{Mbit}$ and $4.67 \mathrm{Mbit}$, respectively, while for $\mathrm{LB}-\mathrm{VHO}$ and $\mathrm{PB}-\mathrm{VHO}$ it reaches 4.08 Mbit and 3.71 Mbit, respectively. Again, these numbers confirm how PLB focuses on goodput maximization and is able to deliver the highest CRBs among the four approaches thanks to the combination of power samples and location information.

From Table 3 we notice that DRI-VHO shows a higher curve for CRBs, with respect to $\mathrm{PB}-\mathrm{VHO}$ and $\mathrm{LB}-\mathrm{VHO}$ (i.e., $26.32 \%$ and $17.32 \%$ higher, resp.) while lower values of CRBs are obtained with respect to PLB-VHO (i.e., 5.84\% lower). In contrast, PLB-VHO presents the highest CRBs trend, that is, (i) $5.98 \%$, (ii) $24.64 \%$, and (iii) $34.17 \%$ higher than DRI, $\mathrm{LB}$, and PB-VHO, respectively. This is justified by the fact that DRI-VHO does not exploit the location information for goodput estimation, then resulting in a higher number of handover attempts and a lower CRBs trend. 
TABLE 3: Statistics for the CRBs for PB, LB, DRI-VHO, and PLB-VHO approaches.

\begin{tabular}{lcccccccc}
\hline $\begin{array}{l}\text { Waiting time } \\
{[\mathrm{s}]}\end{array}$ & $\begin{array}{c}\text { PB mean } \\
{[\text { Mbit] }}\end{array}$ & $\begin{array}{c}\text { PB disp. } \\
\text { index }\end{array}$ & $\begin{array}{c}\text { LB mean } \\
{[\text { Mbit] }}\end{array}$ & $\begin{array}{c}\text { LB disp. } \\
\text { index }\end{array}$ & $\begin{array}{c}\text { DRI mean } \\
{[\text { Mbit] }}\end{array}$ & $\begin{array}{c}\text { DRI disp. } \\
\text { index }\end{array}$ & $\begin{array}{c}\text { PLB mean } \\
{[\text { Mbit] }}\end{array}$ & $\begin{array}{c}\text { PLB disp. } \\
\text { index }\end{array}$ \\
\hline 0 & 3.71 & $49.80 \%$ & 4.08 & $59.58 \%$ & 4.67 & $45.75 \%$ & 4.90 & $38.49 \%$ \\
10 & 3.64 & $48.92 \%$ & 3.94 & $59.46 \%$ & 4.63 & $45.57 \%$ & 4.84 & $37.39 \%$ \\
30 & 3.45 & $48.51 \%$ & 3.71 & $60.53 \%$ & 4.44 & $44.47 \%$ & 4.71 & $38.06 \%$ \\
60 & 3.33 & $48.25 \%$ & 3.44 & $58.90 \%$ & 4.19 & $41.60 \%$ & 4.55 & $35.40 \%$ \\
90 & 3.26 & $45.84 \%$ & 3.53 & $57.28 \%$ & 4.10 & $43.29 \%$ & 4.36 & $44.80 \%$ \\
120 & 3.07 & $47.51 \%$ & 3.34 & $57.29 \%$ & 3.84 & $42.10 \%$ & 4.13 & $44.37 \%$ \\
\hline
\end{tabular}

As a conclusion we can summarize the following results:

(i) PB-VHO makes the lowest number of vertical handovers and provides the lowest CRBs trend (i.e., in the range $[3.07,3.71]$ Mbit). It is suitable for ping-pong effect avoidance, but not for throughput maximization. It does not require high values of waiting time (i.e., $<15$ handovers executed at waiting time $>10 \mathrm{~s})$;

(ii) LB-VHO makes the highest number of vertical handovers and provides a slight increment of CRBs trend (i.e., in the range $[3.34,4.08]$ Mbit). It is suitable for low-QoS services, and the limitation of ping-pong effect occurs only for high values of waiting time (i.e., $<15$ handovers executed at waiting time $>90 \mathrm{~s}$ );

(iii) DRI-VHO makes vertical handovers in the range [10, 79.2] and shows high values of CRBs in the range [3.84, 4.67] Mbit. It is suitable for high-QoS services; its best performance is obtained for high values of waiting time parameter (i.e., $<15$ handovers executed at waiting time $>60 \mathrm{~s})$;

(iv) PLB-VHO makes vertical handovers in the range [8, 53.1] and presents the highest CRBs trend (i.e., in the range [4.13, 4.90] Mbit). It is suitable for highQoS services requiring throughput maximization and limits the ping-pong effect. Its best performance is for medium values of waiting time (i.e., $<16$ handovers executed at waiting time $>30 \mathrm{~s}$ ).

\section{Conclusions}

A novel hybrid vertical handover approach-PLB-VHOfor WLAN and UMTS networks has been presented. It is mainly oriented to ensure service continuity and avoid unnecessary/unwanted handover occurrences. The PLBVHO develops an enhanced location-based approach to build and maintain a power attenuation map, which provides an updated description of the wireless cells in a visited environment. The attenuation map building and update phases are processed by the aid of cooperating mobile terminals within a local area network. RSS samples are then exchanged between mobile nodes, whenever a mobile terminal enters a visited network.

Performance results have been reported to compare PLBVHO technique with a multiparameter vertical handover scheme [11], a traditional power-based [8], and a locationbased [18] vertical handover approach, respectively. We validated the effectiveness of PLB-VHO approach, in terms of (i) a maximization of Cumulative Received Bits and (ii) limitation of the number of vertical handovers. The use of combined location and power information to drive handover decisions brings about goodput enhancements while assuring controlled $\mathrm{VHO}$ frequency with respect to both simple single-parameter, as well as multiparameter approaches, considered in the paper.

\section{Acknowledgments}

The authors are grateful to the anonymous reviewers for their valuable comments and to the journal editor Professor Colonnese for her kind availability. This work was supported in part by RADIOLABS CONSORTIUM.

\section{References}

[1] S. Balasubramaniam and J. Indulska, "Vertical handover supporting pervasive computing in future wireless networks," Computer Communications, vol. 27, no. 8, pp. 708-719, 2004.

[2] G. P. Pollini, "Trends in handover design," IEEE Communications Magazine, vol. 34, no. 3, pp. 82-90, 1996.

[3] J. McNair and F. Zhu, "Vertical handoffs in fourth-generation multinetwork environments," IEEE Wireless Communications, vol. 11, no. 3, pp. 8-15, 2004.

[4] M. Stemm and R. H. Katz, "Vertical handoffs in wireless overlay networks," Mobile Networks and Applications, vol. 3, no. 4, pp. 335-350, 1998.

[5] M. Kassar, B. Kervella, and G. Pujolle, "An overview of vertical handover decision strategies in heterogeneous wireless networks," Computer Communications, vol. 31, no. 10, pp. 2607-2620, 2008.

[6] H. Cho, J. Park, W. Ko, K. Lim, and W. Kim, "A study on the $\mathrm{MCHO}$ method in hard handover and Soft handover between WLAN and CDMA," in Proceedings of the International Conference on Consumer Electronics (ICCE '05), pp. 391-392, January 2005.

[7] K. Ayyappan and P. Dananjayan, "RSS measurement for vertical handoff in heterogeneous network," Journal of Theoretical and Applied Information Technology, vol. 4, no. 10, pp. 989994, 2008.

[8] T. Inzerilli and A. M. Vegni, "A reactive vertical handover approach for WIFI-UMTS dual-mode terminals," in Proceedings of the International Symposium on Consumer Electronics (ISCE '08), pp. 1-4, Vilamoura, Portugal, April 2008. 
[9] S. Xie and M. Wu, "Adaptive variable threshold vertical handoff algorithm," in Proceedings of the IEEE International Conference Neural Networks and Signal Processing (ICNNSP '08), pp. 366-369, Zhenjiang, China, June 2008.

[10] K. Yang, I. Gondal, B. Qiu, and L. S. Dooley, "Combined SINR based vertical handoff algorithm for next generation heterogeneous wireless networks," in Proceedings of the 50th Annual IEEE Global Telecommunications Conference (GLOBECOM '07), pp. 4483-4487, Washinton, DC, USA, November 2007.

[11] A. M. Vegni, G. Tamea, T. Inzerilli, and R. Cusani, "A combined vertical handover decision metric for QoS enhancement in next generation networks," in Proceedings of the 5th IEEE International Conference on Wireless and Mobile Computing Networking and Communication (WiMob '09), pp. 233-238, Marrakech, Morocco, October 2009.

[12] A. M. Vegni, M. Carli, A. Neri, and G. Ragosa, "QoS-based vertical handover in heterogeneous networks," in Proceedings of the 10th International Wireless Personal Multimedia Communications (WPMC 2007), pp. 1-4, Jaipur, India, December 2007.

[13] V. Jesus, S. Sargento, D. Corujo, N. Sénica, M. Almeida, and R. L. Aguiar, "Mobility with QoS support for multiinterface terminals: combined user and network approach," in Proceedings of the 12th IEEE International Symposium on Computers and Communications (ISCC'07), pp. 325-332, July 2007.

[14] A. M. Vegni and F. Esposito, "A speed-based vertical handover algorithm for VANET," in Proceedings of the of 7 th International Workshop on Intelligent Transportation (WIT '10), Hamburg, Germany, March 2010.

[15] F. Esposito, A. M. Vegni, I. Matta, and A. Neri, "On modeling speed-based vertical handovers in vehicular networks "Dad, slow down, I am watching the movie"', in Proceedings of the Annual IEEE Global Telecommunications Conference (GLOBECOM '10), Miami, Fla, USA, December 2010.

[16] S. S. Wang, M. Green, and M. Malkawi, "Adaptive handover method using mobile location information," in Proceedings of the IEEE Emerging Technology Symposium on Broadband Communications for the Internet Era Symposium, pp. 97-101, Richardson, Tex, USA, September 2001.

[17] D. B. Lin, R. T. Juang, H. P. Lin, and C. Y. Ke, "Mobile location estimation based on differences of signal attenuations for GSM systems," in Proceedings of the IEEE International Antennas and Propagation Symposium, vol. 1, pp. 77-80, June 2003.

[18] T. Inzerilli, A. M. Vegni, A. Neri, and R. Cusani, "A locationbased vertical handover algorithm for limitation of the pingpong effect," in Proceedings of the 4th IEEE International Conference on Wireless and Mobile Computing, Networking and Communication (WiMob '08), pp. 385-389, Avignon, France, October 2008.

[19] A. M. Vegni and F. Esposito, "Location aware mobility assisted services for heterogeneous wireless technologies," in Proceedings of the IEEE MTT-S International Microwave Workshop Series on Wireless Sensing, Local Positioning and RFID (IMWS '09), Cavtat, Croatia, September 2009.

[20] W. I. Kim, B. J. Lee, J. S. Song, Y. S. Shin, and Y. J. Kim, "Ping-pong avoidance algorithm for vertical handover in wireless overlay networks," in Proceedings of the 66th IEEE Vehicular Technology Conference (VTC '07), vol. 3, pp. 15091512, September-October 2007.

[21] X. Yan, Y. A. Şekercioğlu, and N. Mani, "A method for minimizing unnecessary handovers in heterogeneous wireless networks," in Proceedings of the 9th IEEE International
Symposium on Wireless, Mobile and Multimedia Networks (WoWMoM '08), pp. 1-5, June 2008.

[22] N. Zhang and J. M. Holtzman, "Analysis of handoff algorithms using both absolute and relative measurements," IEEE Transactions on Vehicular Technology, vol. 45, no. 1, pp. 174-179, 1996.

[23] Y. S. Chen, C. H. Cheng, C. S. Hsu, and G. M. Chiu, "Network mobility protocol for vehicular ad hoc networks," in Proceedings of the IEEE Wireless Communications and Networking Conference (WCNC'09), Budapest, Hungary, April 2009.

[24] M. R. Kibria, A. Jamalipour, and V. Mirchandani, "A location aware three-step vertical handoff scheme for $4 \mathrm{G} / \mathrm{B} 3 \mathrm{G}$ networks," in Proceedings of the IEEE Global Telecommunications Conference (GLOBECOM '05), vol. 5, pp. 2752-2756, St. Louis, Mo, USA, November-December 2005.

[25] X. Yan, Y. A. Şekercioğlu, and S. Narayanan, "A survey of vertical handover decision algorithms in Fourth Generation heterogeneous wireless networks," Computer Networks, vol. 54, no. 11, pp. 1848-1863, 2010.

[26] A. Hasswa, N. Nasser, and H. Hassanein, "Generic vertical handoff decision function for heterogeneous wireless networks," in Proceedings of the 2nd International Conference on Wirelessand Optical Communications Networks (WOCN '05), pp. 239-243, March 2005.

[27] "IEEE 802.21 Media Independent Handover Services-Media Independent Handover," Draft Text for Media Independent Handover Specification.

[28] J. Laiho, A. Wacker, and T. Novosad, Radio Network Planning and Optimisation for UMTS, chapter 3, Wiley, New York, NY, USA, 2nd edition, 2005.

[29] "IEEE Standard for Information technology Telecommunications and information exchange between systems. Local and metropolitan area networks. Specific requirements," Part 11: Wireless LAN Medium Access Control (MAC) and Physical Layer (PHY) Specifications.

[30] J. Laiho, A. Wacker, and T. Novosad, Radio Network Planning and Optimisation for UMTS, chapter 6, Wiley, New York, NY, USA, 2nd edition, 2005.

[31] J. Laiho, A. Wacker, and T. Novosad, Radio Network Planning and Optimisation for UMTS, chapter 3, Wiley, New York, NY, USA, 2nd edition, 2005.

[32] Y. Okumura et al., "Field strength and its variability in VHF and UHF land-mobile service," Review of the Electrical Communication Laboratory, vol. 16, no. 9-10, pp. 825-873, 1968. 

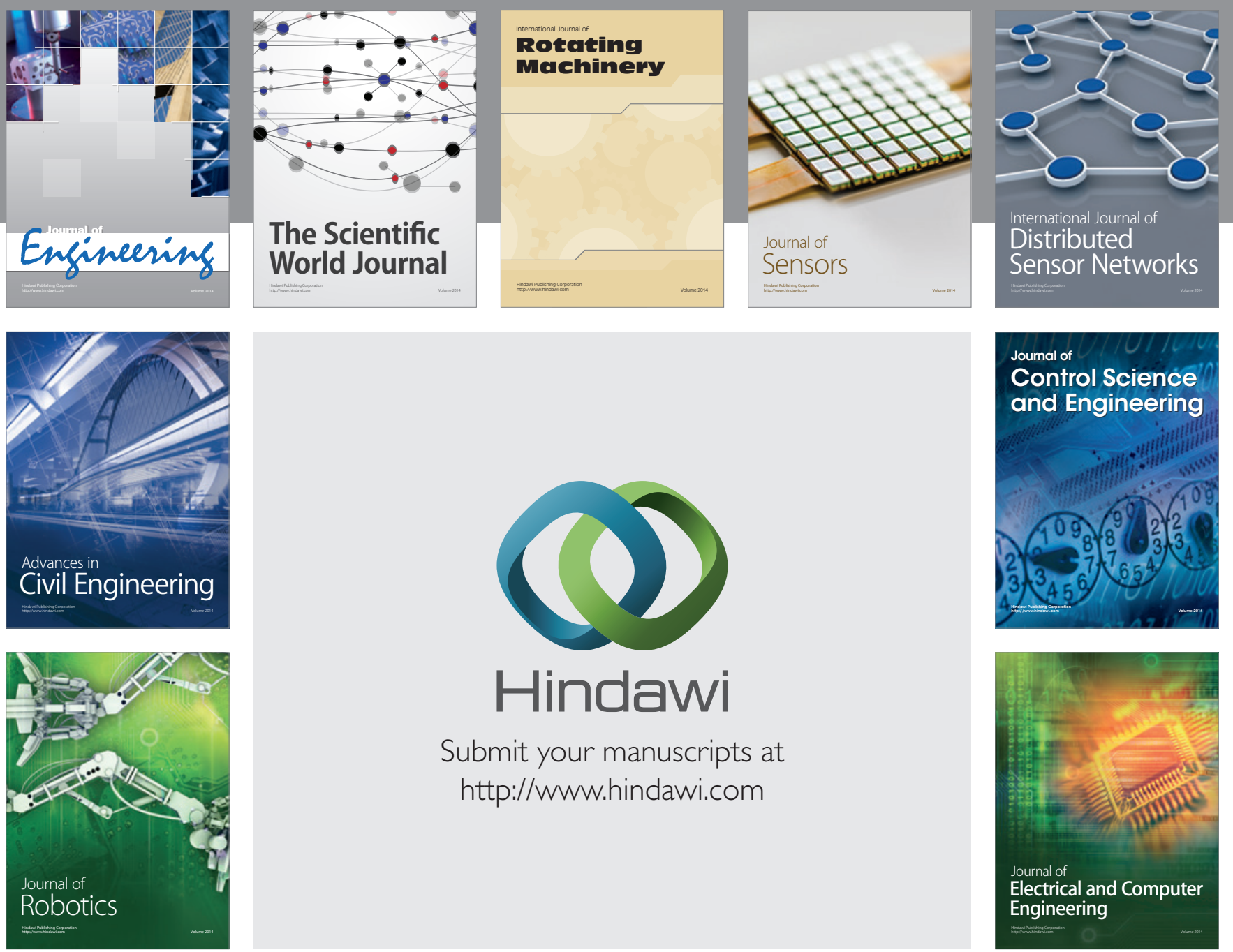

Submit your manuscripts at

http://www.hindawi.com
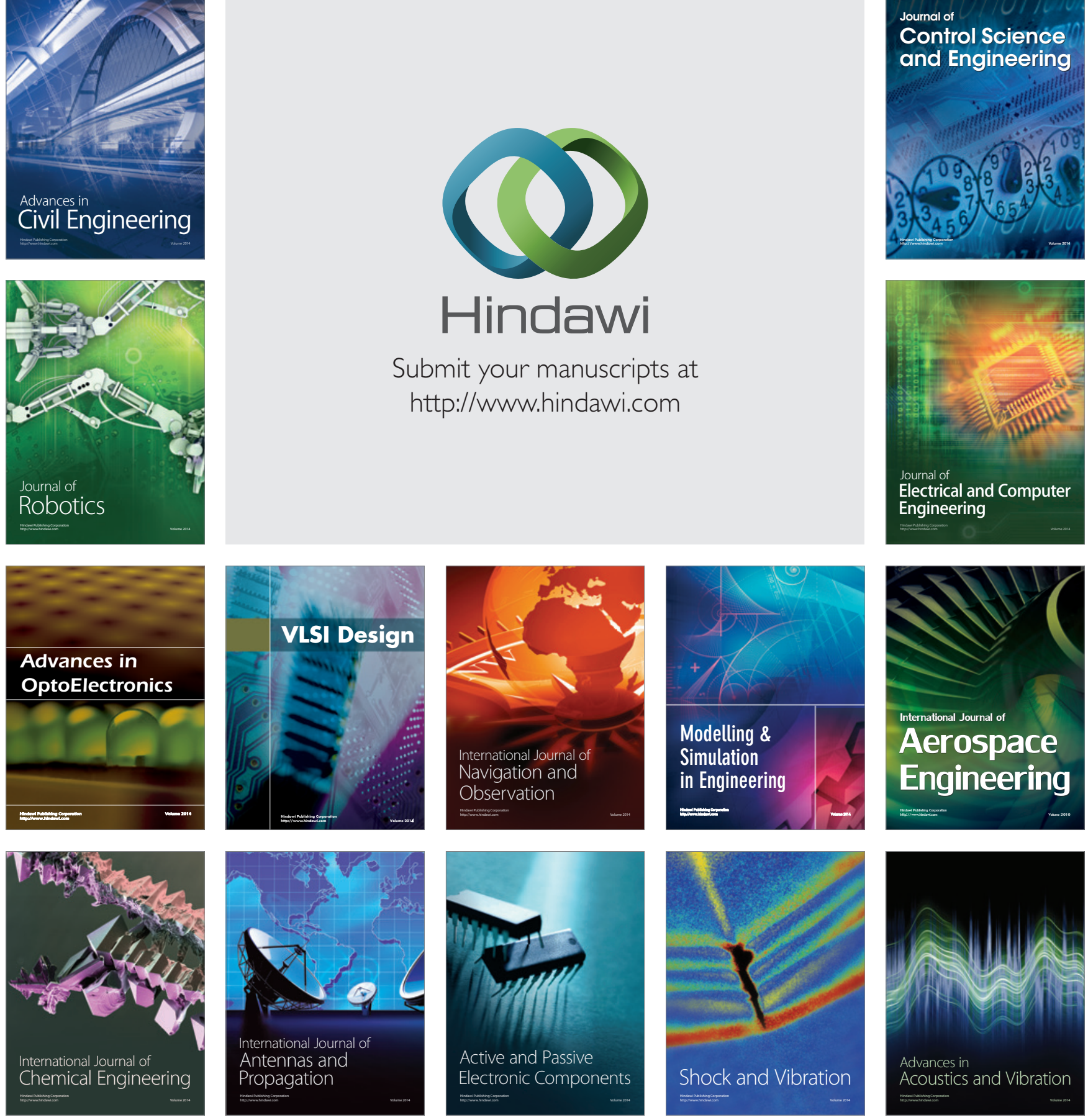